\title{
Análisis del programa de una computadora por niño en instituciones educativas en zonas de exclusión y pobreza: caso Perú
}

\author{
Cerapio Nicéforo Quintanilla Cóndor ${ }^{1}$ (1) @ \\ Juan José Oré Rojas ${ }^{1}$ (D) @ \\ Carlos Rolando Quispe Ccora ${ }^{2}$ (D) (a) \\ ${ }^{1}$ Universidad Nacional de Huancavelica; ${ }^{2}$ Colegio de Alto Rendimiento (COAR), Huancavelica, Perú.
}

Resumen. Este artículo presenta la experiencia en dos instituciones educativas (II. EE) de Educación Básica Regular (EBR), en zonas de pobreza y extrema pobreza de Huancavelica, Perú; poniendo en práctica un programa de una computadora por niño (OLPC). El propósito es valorar el uso de las TIC y su incorporación en el proceso educativo en estos contextos. La metodología es cualitativa: en primer lugar, se realiza el diagnóstico de la situación socioeconómica del contexto para conocer la cosmovisión sobre el uso de las TIC y educación; seguidamente se identificaron las políticas diseñadas por el estado en las II.EE estudiadas y su implementación con relación al desarrollo del currículo nacional. Por último, se realizó la valoración de esta experiencia mediante entrevistas semi estructuradas, registros anecdóticos, observación directa, registros de audio, fotografías y vídeos; cuya información subyacente da cuenta de la experiencia sobre la base de los testimonios y la revisión documental sobre el uso de las TIC. Entre los hallazgos se apunta la necesidad de mejorar la orientación de las políticas para superar la brecha digital, que se mantiene en la zona estudiada, así como las dificultades del enfoque uno a uno (OLPC) en la región.

Palabras clave. Exclusión social; pobreza; educación; TIC.

Análise do programa de um computador por criança em instituições de ensino em zonas de exclusão e pobreza: caso do Peru

Resumo. Este artigo apresenta a experiência em duas instituições de ensino (II.EE) de Educação Básica Regular (EBR), em áreas de pobreza e pobreza extrema de Huancavelica, no Peru; colocando em prática um programa de computador por criança (OLPC). O objetivo é avaliar o uso das TIC e sua incorporação ao processo educacional nestes contextos. A metodologia é qualitativa. Em primeirolugar, realiza-se o diagnóstico da situação socioeconômica do contexto para conhecer a visão de mundo sobre o uso das TIC e a educação. Em segundo lugar, identificam-se as políticas desenhadas pelo Estado nas II.EE estudadas e sua implementação em relação ao desenvolvimento do currículo nacional. Por último, realiza-se a avaliação desta experiência por meio de entrevistas semiestruturadas, registros anedóticos, observação direta, registros em áudio, fotografias e vídeos; cuja informação subjacente explica a experiência com base nos testemunhos e na revisão documental sobre o uso das TIC. Entre as principais conclusões está a necessidade de melhorar a orientação das políticas para superar a exclusão digital que permanece na área estudada, bem como as dificuldades da abordagem 1 a 1 (OLPC) na região.

Palavras-chave: Exclusão social; pobreza; educação; TIC.

Analysis of a One Laptop Per Child program in educational institutions in exclusion and poverty zones: Peru case

Abstract. This article presents the experience in two educational institutions (EEII) of Regular Basic Education (RBE), in areas of poverty and extreme poverty of Huancavelica, Peru, using computers in a One Laptop Per Child (OLPC) program. The purpose is to assess the use of ICT and its incorporation into the educational process in these contexts. The methodology is qualitative: first, it was done the diagnosis of the socioeconomic context to know the worldview about the use of ICT and education. Then, the policies designed by the state in the EEll studied and their implementation in relation to the development of the national curriculum were identified. Finally, the evaluation of this experience through semi structured interviews, anecdotal records, direct observation, audio records, photographs and videos; whose underlying information accounts for the experience based on the testimonies and the documentary review on the use of 
ICT. Among the findings, it is pointed out the need to improve policy orientation to overcome the digital divide, as a situation of educational exclusion that remains in the area studied, as well as the difficulties of the one-to-one approach (OLPC) in the region.

Keywords: Social exclusion; poverty, education; ICT.

\section{Introducción: Diagnóstico de la situación socioeconómica del contexto}

En la Declaración de Incheon, se propuso garantizar una educación inclusiva, equitativa y de calidad, precisando el aprovechamiento de las tecnologías de información y comunicación (TIC) para fortalecer los sistemas educativos y el acceso a la información en una educación de calidad (UNESCO, 2015a).

La globalización ha impulsado en la educación la incorporación de las TIC en sus procesos, con el fin de incrementar su competitividad y así lograr un mejor posicionamiento. La incorporación y masificación de las tecnologías permiten estimular el desarrollo económico, tecnológico y social de las organizaciones y de las naciones. Por lo tanto, es importante conocer y difundir las características básicas y la utilidad de las TIC en el mundo (INEI, 2009).

En las dos últimas décadas, las TIC han evolucionado el funcionamiento de las empresas, los procesos de aprendizaje en estudiantes, las metodologías de investigación en científicos y la manera en que los gobiernos prestan sus servicios a los ciudadanos (INEI, 2009). Es así que, entre las innovaciones más relevantes que se han registrado en la última década de acuerdo a la Meta 13 e Indicador 20 de la OEI, está la incorporación de las $\mathrm{TIC}$ en los procesos educativos, al tiempo que han abierto nuevas posibilidades para la enseñanza y el aprendizaje (OEI, 2016, p. 139).

\subsection{Pobreza y exclusión en la zona de estudio}

El Perú es un país multiétnico, en 2017 registró un total de 31.237.385 habitantes (INEI, 2018b), distribuidos en tres regiones geográficas. La costa, densamente poblada, tiene el $11,7 \%$ del territorio y contiene al $56,3 \%$ de la población. La sierra cubre el $27,9 \%$ del territorio incluyendo al $29,7 \%$ de la población y la selva ocupa el $60,3 \%$ del territorio y alberga al 14,0\% de la población (INEI, 2015b, p. 4). 
En 2014, el gasto real promedio per cápita mensual en Perú fue de S/.646 soles ( $\$ 227,5$ dólares) registrándose un incremento de $0,4 \%$ respecto al año 2013 con una tasa de crecimiento no estable de 5,8\% (INEI, 2015d); sin embargo, en 2013, la desigualdad fue muy alta con un índice Gini de 0,447 (Banco Mundial, 2015); entre 2013 y 2014, la línea de pobreza extrema se incrementó en 3,7\% a nivel nacional (INEI, 2015c, p. 29), equiparable a la canasta familiar (año 2015) de S/.161 soles mensuales ( $\$ 49$ dólares) por integrante del hogar. La incidencia de la pobreza en zona rural es alta, con un 46,0\%, mientras que en el área urbana la tasa de pobreza es 15,3\% de la población (INEI, 2015c). En 2017, el Perú presenta una reducción de la pobreza monetaria en un $21,7 \%$, aunque persisten tasas altas de pobreza en zonas rurales. Huancavelica ocupa el segundo lugar en pobreza con tasas que se ubican entre el intervalo de 33,3\% y 36,8\% (INEI, 2018a).

\subsection{Localización geográfica}

Ni la pobreza rural ni el aislamiento poblacional han sido materia de estudio sistemático en el Perú, cuando "las carreteras y los puentes son el mejor instrumento de inclusión contra la pobreza” (Webb, 2013, p. 15). En el Perú, específicamente en nuestro contexto de estudio, las zonas rurales de Huancavelica, existen centros poblados que no cuentan con conectividad de carreteras por estar ubicados en lugares de difícil acceso. El obstáculo geográfico debe entenderse tanto por su acción directa, a través de los costos de comercialización y de acceso a información, como por su acción indirecta, cuando contribuye a las deficiencias de educación, salud, tecnología, fuerza política, infraestructura, capital social y otros elementos que restringen la capacidad productiva (Webb, 2013).

\subsection{Acceso a medios de comunicación}

El grado de aislamiento en el Perú se redujo gradualmente a lo largo del siglo XX por efecto de una expansión gradual de la red vial y de una lenta y limitada aparición del teléfono fijo (Webb, 2013); sin embargo, en algunas zonas rurales de difícil acceso de la sierra y costa, tuvo mayor acercamiento de conectividad a partir de la primera década del siglo XXI.

En el censo 2017, se registró un total de 8.352.284 de viviendas con ocupantes presentes; de los cuales, 7.101.717 hogares utilizan al menos una TIC $(86,1 \%)$ (INEI, 2018b). En la tabla 1 se muestra el detalle del acceso en la región de Huancavelica. 
Tabla 1. Hogares con acceso a las TIC en Perú y Huancavelica según censo 2017

\begin{tabular}{cccccccc}
\hline \multicolumn{2}{c}{ Internet } & \multicolumn{2}{c}{ Teléf. móvil } & \multicolumn{2}{c}{ Teléf. fijo } & \multicolumn{2}{c}{ Tv. cable } \\
\hline Perú & Hvca & Perú & Hvca & Perú & Hvca & Perú & Hvca \\
$28,0 \%$ & $4,9 \%$ & $83,8 \%$ & $68,2 \%$ & $21,9 \%$ & $2,0 \%$ & $37,6 \%$ & $7,2 \%$
\end{tabular}

Fuente: Instituto Nacional de Estadística e Informática 2018b

La mayoría de los hogares que no tienen acceso a las TIC están en los departamentos de Loreto $(32,3 \%)$, Huancavelica $(31,5 \%)$ (zona del estudio), Amazonas (29,7\%), Puno (28,4\%) y Apurímac (28,3\%) (INEI, 2018b).

La penetración en las zonas rurales del teléfono celular, la televisión por cable, más el acceso a cabinas de Internet en los pequeños pueblos, constituyen un salto a la conectividad (Webb, 2013). Sin embargo, Huancavelica es una región con alto porcentaje de zonas rurales (79\%) con mayor pobreza multidimensional (70,66\%) (Vásquez Huamán, 2012); sus centros poblados se encuentran aislados y desconectados, sus habitantes son quechua hablantes con una tasa de $17,7 \%$ de analfabetismo (INEI, 2018b).

\subsection{La inversión educativa en el Perú}

El Perú invierte en educación \$236 dólares por alumno al año, mientras que Suecia $\$ 4.050$ dólares, Estados Unidos $\$ 2.478$ dólares, Finlandia $\$ 3.404$ dólares y Noruega, que es el país que más invierte, $\$ 6.758$ dólares (Expansión, 2015). En el reporte del BID para el año 2018, según CIRCA 2016, Perú hace inversión pública en educación de sólo el 3,8\% del PBI (Jaureguiberry, López y Zoido, 2018).

La tasa de matrícula más alta se registra en educación primaria con un 92,1\%, y el nivel de educación secundaria tiene 83,1\% (INEI, 2015a). Del total de alumnos matriculados, las zonas rurales registran bajas tasas de matrícula, solo el $36,2 \%$ en el nivel de educación primaria y el $32 \%$ en educación secundaria.

Los resultados de la Encuesta Nacional de Hogares del año 2012, muestra que el $5,9 \%$ de la población de 25 y más años de edad no logró estudiar ningún nivel de educación, el 26,9\% alcanzó estudiar algún grado o año de educación primaria, el 36,3\% algún año de educación secundaria y el 30,9\% educación superior (no universitaria o universitaria) (INEI, 2014).

En el año 2000, la tasa mundial de analfabetismo registró una leve reducción de un $18 \%$ y en el año 2015 un estimado de 14\% (UNESCO, 2015b, p. 8), de ellos, el $64 \%$ son mujeres. Perú tiene índices más bonancibles. Los resultados del Censo del 2017, revelan que existen 1.262.075 personas de 
15 a más años de edad que no saben leer ni escribir, representa el 5,8\% de la población, de los cuales el $8,5 \%$ son mujeres y $3,1 \%$ son varones; además el $17 \%$ se encuentra en zona rural y el $3,2 \%$ en zona urbana (INEI, 2018b). Huancavelica registra alta incidencia de analfabetismo (17,7\%); debido a la escasa inversión en educación (Ñopo, 2018), demostrando que no existe una prioridad dentro del presupuesto público. Sin embargo, en este contexto, se ha iniciado una política de centros educativos de excelencia, denominados colegios de Alto Rendimiento (COAR), con marcada diferencia respecto al estándar de la educación peruana.

\section{Las TIC y las políticas educativas en el Perú}

En los últimos cinco años, se reconoce que América Latina es la región más proactiva del mundo en relación con la integración de las TIC en sus sistemas educativos, contribuyendo de esta manera a la inclusión social, la democratización y la reducción de la brecha digital (SITEAL, 2014, p. 32). La brecha digital en el continente hace referencia a tres aspectos concurrentes: brecha en el acceso a las TIC, brecha en el uso de las TIC, y brecha en las expectativas (SITEAL, 2014, p. 33).

Los conocidos como "modelo 1:1" o un "ordenador por niño" (en la bibliografía internacional "modelo 1 a 1", "1:1", "computación ubicua en las escuelas", o "inmersión tecnológica") (1:1 model; ubiquitous; technology immersion) son experiencias que vienen cobrando relevancia incluyendo a los países altamente desarrollados (Area, 2011, pp. 52-53). Brasil en el 2007, inició el programa Uno a Uno llamado Um Computador por Aluno (UCA) y PROINFO; también en Paraguay, con la Fundación Paraguay Educa; en Uruguay, el Plan Ceibal el más grande del mundo; en Colombia en el 2008 el programa Colombia aprende y computadoras para educar. En el 2009, Chile lanzó el programa Laboratorio Móvil Computacional (LMC) y Argentina en el 2010 con el programa "Conectar Igualdad"; el mismo año, Ecuador lanza el programa Mi Compu (Severin y Capota, 2011); luego en México primero con Red Escolar, luego, con Enciclomedia y, finalmente, implementando Habilidades Digitales para Todos (SITEAL, 2014).

En el caso del Perú, existen iniciativas sobre el uso de tecnologías educativas desde el año 1996 con la robótica educativa, en el 2001 con el Plan Huascarán y la incorporación de las XO, programa 1 a 1 en el año 2007.

En 1996, se ponen en marcha dos programas de tecnología educativa en las escuelas públicas peruanas: El Programa EDURED, de la Unidad de Redes Educativas (Peralta y Lugo, 2016); y el Proyecto de robótica escolar 
denominado INFOESCUELA, que integraba el Programa de Mejoramiento de la Calidad de la Educación Primaria (MECEP). En cuanto a la modalidad de la Educación a Distancia (EDIST), ésta se incorporó al Proyecto Huascarán en el 2002, sin embargo luego de dos años, el Programa EDIST volvió a ser independiente del Proyecto Huascarán (Balarín, 2013); finalmente en el año 2007, la Dirección General de Tecnologías Educativas (DIGETE) del MINEDU lanzó el proyecto Una Laptop por Niño (ULPN).

\subsection{Proyecto Huascarán}

Mediante Decreto Supremo 067-2001-ED, se creó el Proyecto Huascarán con el fin de mejorar la calidad educativa en las zonas rurales y urbanas del país a través del uso de las TIC en EBR (MINEDU, 2001). EI Portal Educativo Nacional (http://huascaran.edu.pe no disponible) fue el entorno de aprendizaje virtual que el proyecto Huascarán brindó a la comunidad educativa. En él se encontraron módulos de ciencia, videos educativos, libros digitales, diccionarios políglotas, plataformas virtuales basadas en Moddle, y servicios de comunicación, tales como foros y Boletín Pedagógico Huascarán, permitiendo la conformación de la Comunidad Virtual Huascarán (MINEDU, 2006).

Este proyecto tuvo interconexiones entre las instituciones y comunidades educativas involucradas, realizadas mediante un centro operador telefónico y una señal satelital (plataforma tecnológica satelital hub) dirigidas desde el data center y el satélite Panamsat 1-R enlazando estaciones remotas VSAT (450 estaciones) instaladas en todo el país (MINEDU, 2006).

Los resultados de este proyecto se reflejan en la tabla 2.

Tabla 2. Cifras del proyecto Huascarán

\begin{tabular}{lrrrrrrr}
\hline \multicolumn{1}{c}{ Concepto } & $\begin{array}{c}\text { Ago-dic } \\
2001\end{array}$ & 2002 & 2003 & 2004 & 2005 & $\begin{array}{c}\text { Ene-abr } \\
2006\end{array}$ & Total \\
\hline $\begin{array}{l}\text { Instituciones educativas } \\
\text { (II.EE) beneficiadas }\end{array}$ & 6 & 1098 & 1099 & 503 & 310 & 4 & 3020 \\
$\begin{array}{l}\text { Estudiantes beneficiados } \\
\text { Docentes capacitados }\end{array}$ & 5704 & 1224477 & 1006113 & 111342 & 179409 & 619 & 2527664 \\
$\begin{array}{l}\text { Computadoras adqui- } \\
\text { ridas }\end{array}$ & 60 & 10813 & 6767 & 21096 & 14437 & 2338 & 55451 \\
$\begin{array}{l}\text { Antenas para Internet vía } \\
\text { satélite adquiridas }\end{array}$ & 0 & 0 & 0 & 4020 & 5613 & 0 & 14806 \\
Conexiones a Internet & 0 & 113 & 123 & 845 & 424 & 145 & 1650 \\
\hline
\end{tabular}

Fuente: MINEDU 2006 
Este ambicioso proyecto no logró la sostenibilidad necesaria como política educativa de los gobiernos del Perú.

\subsection{Las X0 en el Perú}

La Dirección General de Tecnologías Educativas (DIGETE) del MINEDU, y como parte del proyecto Huascarán, Ianzó en 2007 el proyecto ULPN, con el propósito de dotar computadoras portátiles XO a estudiantes y docentes de escuelas de educación primaria (prioritariamente unidocentes) de áreas rurales en extrema pobreza, utilizadas como herramientas pedagógicas para mejorar la calidad educativa y acortar la brecha digital con las áreas urbanas.

En 2013, solo el 30\% de las II.EE contaban con servicio de Internet, así como las XO distribuidas de 9 a 30 computadoras por I.E. Este mecanismo contradice el modelo 1 a 1 , porque en cada salón de clases hay más estudiantes que XO. Además, señala que el Programa ULPN fue mencionado por primera vez en la Ley del Presupuesto General de la República del 2007; pero no se encontró documentos, sistematización, ni registro en el Sistema Nacional de Inversión Pública. En consecuencia, se considera como una actividad o iniciativa y no como un Programa (OLPC News, 2012).

Entre los años 2005-2010, se adquirieron un poco más de 850.000 laptops XO, distribuidas de la siguiente manera: en primaria 600.000 unidades, atendiendo a 9.400 escuelas unidocentes con 180.000 niños y en secundaria un total de 250.000 unidades, destinadas al Centro de Recursos Tecnológicos (CRT), para 6.700 colegios, 1.800 .000 estudiantes y 100.000 maestros. Cabe resaltar que, en la segunda etapa del proyecto, se abandonó el modelo 1:1 por falta de recursos y se optó entonces por entregar una laptop por cada diez estudiantes (Balarín, 2013).

La iniciativa ULPN en el Perú fue un proyecto de gobierno ejecutado por MINEDU, no siendo una política nacional que garantice su continuidad en gobiernos siguientes, implementándose sin estudio ni planificación (OLPC News, 2011). En general, a este Programa se le observaron debilidades en cuanto a las mejoras que el acceso a las TIC podría traer a la educación peruana. El uso político, sin planeamiento, evidenció la entrega de computadoras en forma desigual; además de indicios de corrupción en los procesos de compra de bienes y concesiones (Balarín, 2013, p. 19). 


\subsection{La robótica en el Perú}

La robótica en las II.EE públicas del Perú se inicia en 1996. La Dirección Nacional de Educación Inicial y Primaria, del MINEDU, fue la encargada de administrar el proyecto INFOESCUELA. La aplicación piloto integró 12 II.EE públicas ( 6 en Lima Metropolitana y 6 en otras regiones); con algunas particularidades, en Ayacucho, los comandos del Ienguaje de programación LOGO fueron diseñados en quechua, garantizando el aprendizaje de ciencias, matemáticas y tecnología en su lengua materna (Linares Gallo, 2015). Las pruebas pilotos se desarrollaron entre 1997 y 1999 con resultados satisfactorios, bajo la supervisión del profesor Seymour Papert; y luego, por miembros de la Universidad de Hartford, quienes sugieren la estandarización del material Lego.

En 2001, ya con 500 escuelas involucradas con la robótica educativa, se vincula la experiencia de INFOESCUELA con LEGO al proyecto Huascarán en la educación primaria. En 2008, el MINEDU solicitó la asistencia del BID para desarrollar y validar un enfoque pedagógico para el Área de Ciencia y Ambiente del tercer grado de primaria el cual fue aceptado (Linares Gallo, 2015).

En 2010, el MINEDU adquirió Módulos de Robótica Educativa para implementar los CRT y Aulas de Innovación Pedagógica del ULPN, con el objetivo de mejorar la calidad de la educación (priorizando el área de ciencias naturales. La adquisición comprendió 20.000 licencias de Robótica WeDo con configuración del laptop XO (versiones 1.0 y 1.5) y 128.000 kits de Robótica Educativa WeDo. En Huancavelica en 2011, se implementó con 3.379 Kits de equipos robóticos, distribuidos en $811 \mathrm{II}$.EE de educación primaria; y en secundaria 8.380 para 271 II.EE (Quintanilla, Cayllahua y Canales, 2013).

En 2016, el director de Innovación Tecnológica en Educación del MINEDU, Jorge Peralta prometió, sin llegar a concluir, la entrega de 25.000 tablets y 42.000 kits de robótica con el fin de promover el uso de la tecnología a favor del aprendizaje, así como potenciar el pensamiento crítico y el trabajo en equipo. Las tabletas serán para escolares de quinto y sexto de primaria de 288 II.EE en 17 regiones, donde actualmente se desarrolla la estrategia de Soporte Pedagógico (El Peruano, 2016). 


\section{Valoración de las experiencias educativas en el uso de las TIC}

El análisis de las experiencias que presentamos en este estudio obedece al enfoque cualitativo. Su propósito no es generar una explicación generalizada a todas las escuelas de América Latina, sino comprender un fenómeno específico (Creswell, 2012) mediante la reflexión sobre el uso de las TIC y la participación de las familias de dos escuelas ubicadas en zonas rurales de extrema pobreza; la primera, del nivel educativo secundario en la comunidad de Llahuecc y la segunda, del nivel educativo primario en la comunidad de Chuñuranra, ambos en la provincia de Huancavelica, Perú.

En el primer momento se realizaron visitas de campo para dar inicio al diagnóstico y conocer la situación socioeconómica de los sujetos de estudio, tal y como se ha reflejado al principio. Posteriormente se procedió a la aplicación de los instrumentos de recolección de información: Entrevista semi estructurada, documentos fotográficos, videos, registros anecdóticos sin dejar de lado la observación directa que nos permitiera llegar a la valoración y reflexión sobre la experiencia presentada.

Para el análisis de datos, se transcribieron y tradujeron las entrevistas respetando la interculturalidad de los sujetos de estudio en cuanto al uso de su lengua originaria. La decodificación de la información que subyace en los documentos fotográficos y videos fueron de gran importancia para valorar la experiencia que a continuación presentamos.

Se realizaron informes densos que integran la información recogida en cada escuela. Se compartieron con los interesados para su valoración.

\subsection{Experiencia: I.E José A. Encinas Franco de Llahuecc}

Llahuecc es un pueblo muy pequeño integrado por 50 familias, localizado a $3.780 \mathrm{msnm}$, en el distrito de Acoria, provincia de Huancavelica; el traslado desde Huancavelica, en camioneta rural, es de dos horas y 25 minutos desde Acoria; el camino es muy agreste y accidentado (figura 1). Sus habitantes son quechua hablantes de extrema pobreza; se dedican a la agricultura y ganadería de sustento familiar. 


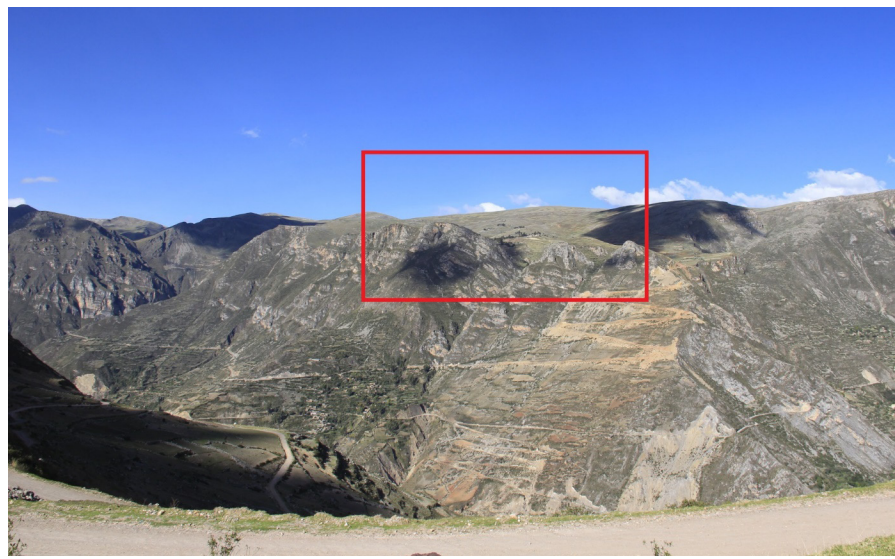

Figura 1. Localización de Llahuecc, Acoria, Huancavelica.

Fuente: Archivo de los autores.

La I.E Mx. José A. Encinas Franco está ubicada en la parte central de la comunidad, colinda con el campo deportivo, delimitada por un cerco de tapial deteriorado; tiene patio a cielo abierto con piso de tierra; cuenta con dos pabellones; el primero, tiene dos aulas inadecuadas (pared de tapial, techo de calamina, cielorraso de triplay, de $4 \mathrm{~m}$ por $5 \mathrm{~m}$, piso de tierra, carpetas deterioradas); y el segundo tiene 5 aulas adecuadas (pared de tapial, techo de calamina, cielorraso de triplay, de $5 \mathrm{~m}$ por $5 \mathrm{~m}$, carpetas unipersonales), de las cuales, una ocupa la Dirección del Colegio y otra la sala de Innovación Pedagógica. Es de hacer notar que la I.E no cuenta con sala para profesores, jefaturas de estudios, comedor, laboratorio, sala para padres de familia, aulas para arte y música para el desarrollo de actividades escolares, como se observa en las figuras 2.

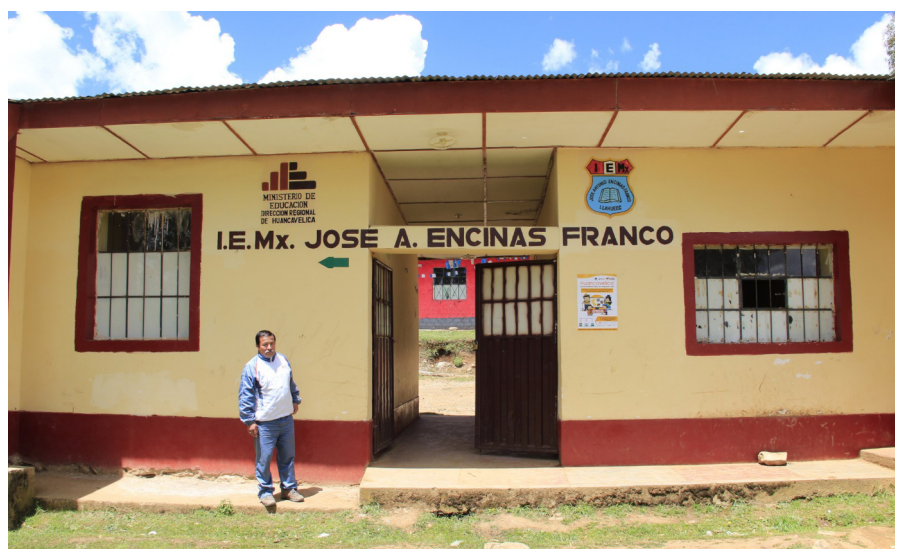

Figura 2. Edificio principal de la escuela I.E Mx. José A. Encinas Franco de Llahuecc, Acoria, Huancavelica Fuente: Archivo de los autores. 
Asimismo, se observa que no cuenta con ambientes para servicios de aseo, solo dispone de cuatro silos (pozos ciegos) ubicados dos al sur y dos al norte, con dos compartimentos cada uno (figura 3).

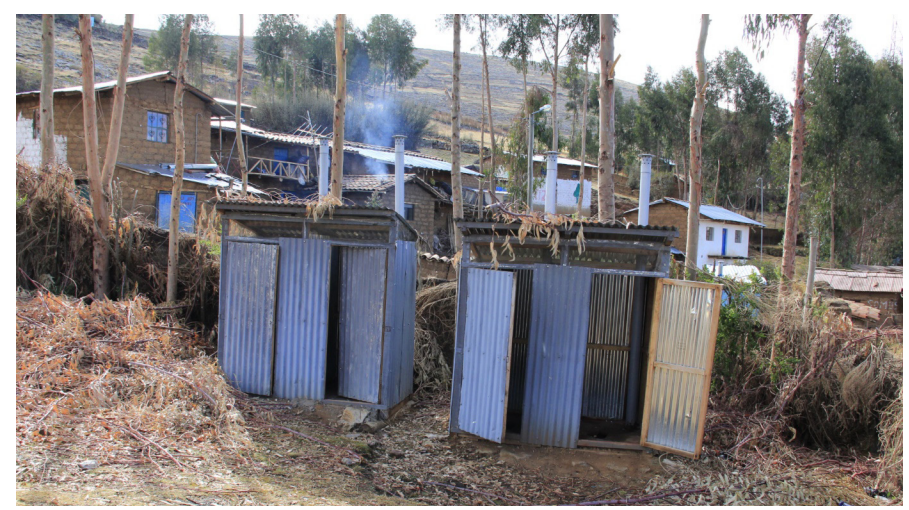

Figura 3. Letrinas de la I.E Mx. José A. Encinas Franco de Llahuecc, Acoria, Huancavelica.

Fuente: Archivo de los autores.

La Institución está a cargo de una directora, cuenta con 9 profesores: 5 nombrados (permanentes) y 4 contratados (anualmente). Su matrícula escolar es de 65 estudiantes procedentes en su mayoría de Llahuecc, y de comunidades vecinas de 2 a $5 \mathrm{~km}$ de distancia; quienes se trasladan a pie por caminos agrestes y accidentados, no transitables con bicicletas donadas por el gobierno.

Los profesores no son del lugar. Generalmente se integran a la I.E durante 4 días, a excepción de los contratados; viven en habitaciones precarias a corta distancia de la I.E, organizándose solidariamente para preparar sus alimentos.

Las clases se desarrollan en las mañanas; por las tardes los profesores planifican actividades escolares, en algunos casos brindan apoyo a los niños y niñas que asisten al colegio a realizar sus tareas.

\subsubsection{Las TIC en la Institución Educativa}

La institución educativa cuenta con un aula de "Innovación Pedagógica" donde desarrollan las clases de computación, sirve como depósito de materiales educativos (computadoras, libros, bicicletas, instrumentos musicales), biblioteca y sala de lectura (figura 4). No cuenta con Internet de banda ancha por fibra óptica; se recibe a través de una antena parabólica de baja velocidad (figura 5). Los libros se encuentran apilados en las ventanas 
y algunos estantes. El responsable de la administración de este espacio es la directora quien atiende a los estudiantes de acuerdo a su disponibilidad de tiempo.

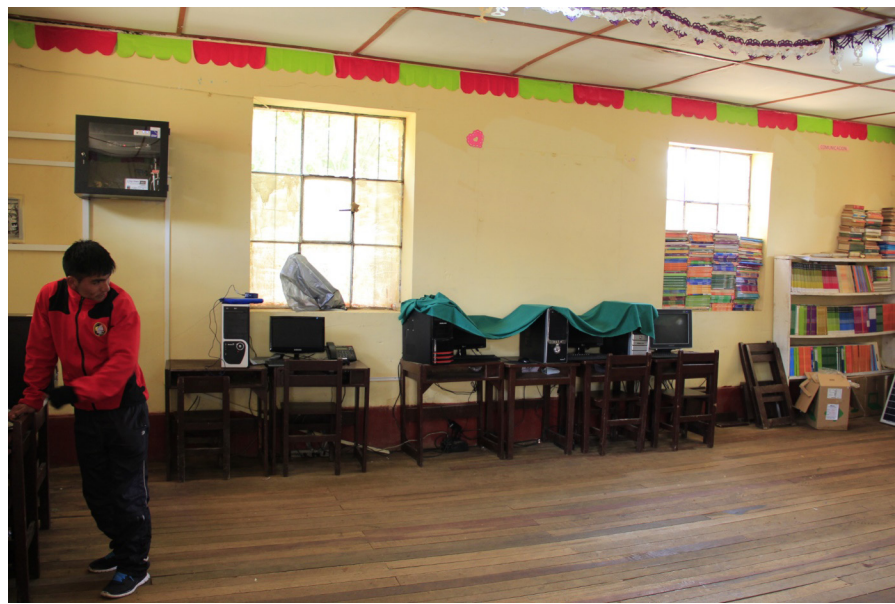

Figura 4. Aula de innovación pedagógica de la I.E Mx. José A. Encinas Franco de Llahuecc, Acoria, Huancavelica.

Fuente: Archivo de los autores.

El uso de las tecnologías en esta I.E nace con el Proyecto Huascarán, sin llegar a su implementación. En 2011, recibe 25 computadoras XO y un equipo de panel solar para su funcionamiento. Durante las visitas se observó que las XO se encuentran almacenadas en cajas, los estudiantes y profesores las usan raras veces.

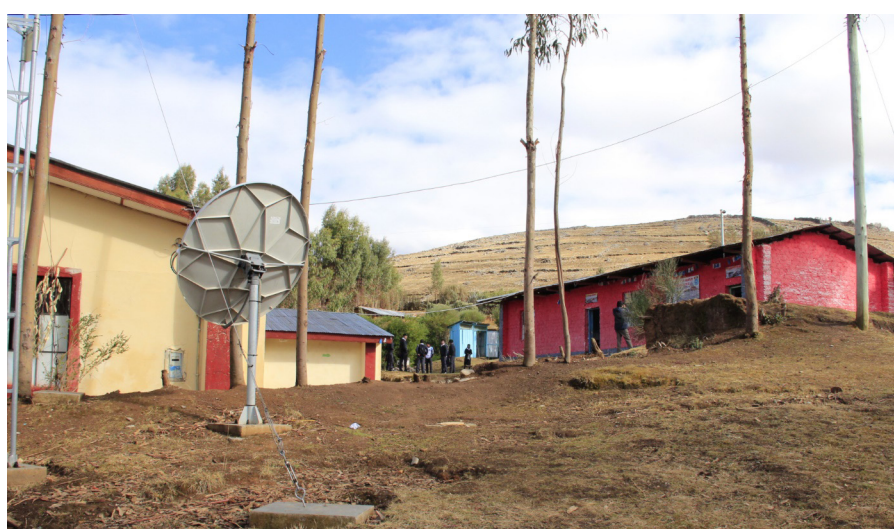

Figura 5. Antena parabólica para internet de la I.E Mx. José A. Encinas Franco de Llahuecc, Acoria, Huancavelica.

Fuente: Archivo de los autores. 
Llama la atención la participación de la Asociación de Padres de Familia (APAFA) quienes se organizaron para la compra de seis computadoras en el periodo escolar 2012 - 2013 de plataforma Windows XP, con el fin de que sus hijos tengan acceso al uso de esta tecnología que consideran más amigable; de estos equipos aún hay cuatro que están en buen estado.

\subsubsection{Las TIC y los profesores en el aula}

Con miras a lograr una mejor calidad de vida de las poblaciones vulnerables la OEA (2014) considera que, en espacios donde los marginados y excluidos requieren mayor apoyo, se debe tener en cuenta el ecosistema más amplio de la tecnología que permita a través de la educación alcanzar la equidad. Además, es necesario elegir tecnologías adecuadas que les permitan a los profesores fortalecer sus competencias básicas de hardware y software, y el uso pedagógico de las tecnologías (software, programas, multimedia, video, Tv y radio) acorde al currículo de su área.

Los profesores de la escuela no tienen dominio en el uso de las TIC. Así se manifiestan algunos de ellos:

Profesor A (de ciencias sociales): ... "conocen lo básico, no son expertos, no están altamente capacitados, o sea lo básico... así como yo ..., solo manejamos algunos programas como el Word, Excel y Power point"

Profesor B (de informática): ... “los estudiantes se aburren con las clases tradicionales, en cambio con las computadoras se motivan por encontrar animaciones, figuras y otras. Les gusta ver videos educativos gracias al internet ellos tienen mucha información"

... "algunos profesores de algunas áreas hacen uso de Internet; son de las áreas de sociales, biología, y otras más, la información lo tienen en Internet, usan la información o bajan en su USB para realizar sus clases"

Tampoco tienen formación básica. Los profesores manifiestan lo siguiente:

Profesor B: ... "en cuanto a las XO tengo poco dominio, conozco un poco de Scratch, Turtle Art y Etoys; más desarrollamos ofimática con software libre"

Profesor C: ... "soy profesor de Ciencias Naturales, no tuve otra opción de aceptar desarrollar la asignatura, porque los demás profesores no tienen conocimiento sobre el uso de Internet y computación. Tengo formación de ingeniero en agronomía" 
Se observa la debilidad en la formación de competencias de los profesores para el uso de las TIC en las asignaturas acorde a estándares en TIC propuestas.

También se constató en las clases visitadas. El proceso de enseñanza aprendizaje se desarrolla de manera tradicional (figura 6). Además, no cuenta con un horario establecido para el uso del aula de innovación.

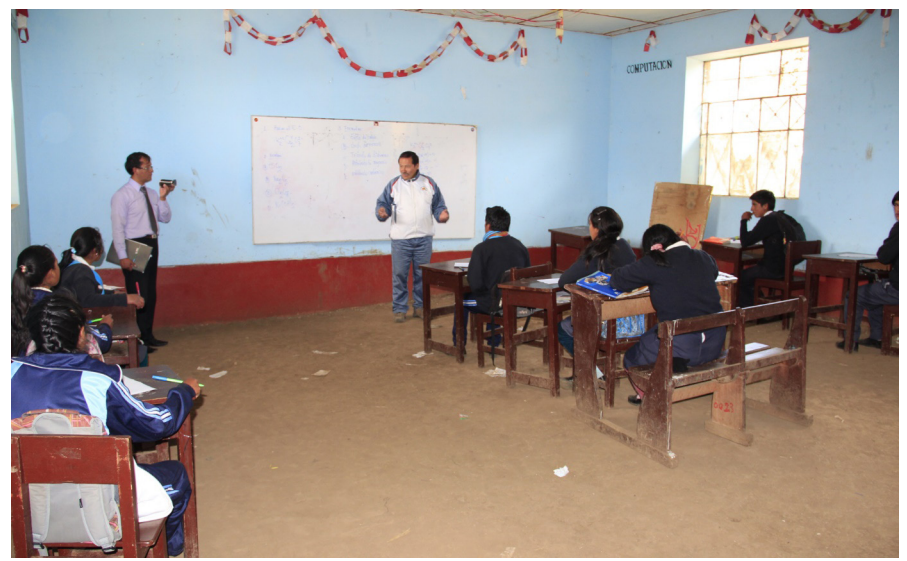

Figura 6. Una clase en la escuela I.E Mx. José A. Encinas Franco de Llahuecc, Acoria, Huancavelica.

Fuente: Archivo de los autores.

Esto en parte es así porque, en Perú no existe normativa para el uso de las TIC en el proceso educativo, solo es considerado como eje transversal en el Diseño Curricular Nacional (DCN) (MINEDU, 2008).

Una estudiante corrobora el tipo de usos con TIC en las aulas.

Hija: "El profesor cada vez que entramos a la sala de cómputo nos indica qué vamos a hacer en la computadora y el que termina más rápido tiene mejor nota. En cuanto a las clases de lenguaje el profesor realiza solo en la pizarra; en ciencias sociales, algunas veces su laptop y nos muestra videos; el de matemática solamente pizarra. Solo en las clases de computación entramos en las computadoras, trabajamos en Power Point, Ponemos texto, le ponemos figuras, lo adornamos. También hacemos algo de Excel".

Sin embargo, en reunión de profesores se determinó que los estudiantes deben desarrollar capacidades en el uso de las tecnologías en la asignatura de Educación para el Trabajo, orientados a promover microempresas; con el fin de insertar a los estudiantes en el mercado laboral. 
Al respecto, se observó que los profesores de informática desarroIlan temas relacionados a Ofimática y contenidos que involucran actividades para diseñar ediciones de video y fotografía, sin considerar los contenidos propuestos en el currículo.

Con todo, el entusiasmo por estas clases y sus posibilidades es muy grande. El testimonio de la directora así lo indica:

... "Ios papás están felices, porque quieren que sus hijos sepan computación, como es zona rural... "hasta bajan música por internet; aunque nuestro Internet es muy lento... antes la educación era tradicional del bla bla bla... y ahora se da el uso de la tecnología... por ejemplo, los chicos decían que no sabían bailar danza moderna, viendo el video en Internet han aprendido a bailar"

Asimismo, el profesor de informática manifiesta:

... "Ios padres tienen mucho interés que sus hijos aprendan computación, porque es importante para el trabajo... hay niños que no son del lugar y se quedan hasta las 15 horas porque tienen preferencia para hacer uso de las computadoras, luego los del lugar vienen en las tardes porque también necesitan aprender. En el colegio solo hay una niña que tiene Tablet"

Es notorio el interés que muestran los estudiantes por el uso de las TIC a pesar de las limitaciones existentes con relación a la cantidad de equipos disponibles, la falta de integración en el currículo, la falta de formación de los docentes y la baja calidad en el acceso al servicio de la red.

\subsubsection{Participación de la familia en el uso de las TIC}

El testimonio de una madre de familia revela el esfuerzo que hace para enviar a sus hijos al colegio.

..."hace dos años mis hijas estudiaban en Acoria, pero es muy lejos, caminaban mucho casi tres horas. Entonces ellas llegaban tarde y cansado a la casa. Ya ni querían estudiar"

... "mis hijas tuvieron que caminar. Tenía que levantar casi muy oscuro en la mañana, Ilorando ella a veces se levantaba. Llegaba tarde al colegio y los profesores no le entendían, le ponían falta o tarde... me decían que ya se les va a dar una bicicleta y nada... por eso le puse en el colegio de aquí arriba (I.E. José Encinas Franco), está a una hora, lo malo es que es un poquito bajo la enseñanza. Por eso me preocupa, el día que termine su secundaria, ¿rendirá, cómo será?" 
La madre informante es jefa de familia, su esposo trabaja lejos

... "cuando había reunión en Acoria iba muy temprano, amarro mis dos vaquitas y asisto a la reunión hasta las 2 o las 3 de la tarde... así también mis hijos mayores, estudiaron en Acoria... mi esposo se fue a trabajar muy lejos, regresa cada medio año con eso puedo educar a mis hijas"

La realidad estudiada refleja que para las familias en extrema pobreza es difícil educar a sus hijos, las condiciones económicas no son alentadoras.

Siguiendo la indagación en el uso de las TIC, esta vez en el contexto de las familias, se concertó la visita a la casa de la niña que, por referencia del docente, dispone de Tablet, constatándose lo siguiente:

Madre: Nosotros somos muy pobres y no podemos comprar, no tenemos lo que dices Tablet. Yo, cuanto quisiera tener para mis hijos.

Entrevistador: ¿Llega señal de televisión por acá? (existe una antena parabólica en el techo la casa)

Madre: No hay señor, mis hijos cuando vivían aquí han hecho poner cable, pero como ya se fueron, solamente quedó la antena "no tenemos para pagar", mis hijitos a veces me mandan dinero para nuestro gastito y para mi hija que estudia.

Entrevistador: Cómo en la zona no hay teléfono fijo, ¿Usted y su hija tienen teléfono celular?

Madre: Si tengo solamente una, porque mi hijo me regaló para que me llame; es muy pequeño. Tampoco no sé su precio ni cuánto vale.

Asimismo, se mantuvo una conversación con la niña.

Entrevistador: ¿usas el celular de tu mamá para Facebook? (induciendo el uso de redes sociales).

Hija: Yo sí, a veces lo utilizo en Ilamadas a mis hermanas. El celular es pequeño y no tiene para Facebook.

Entrevistador: ¿tienes Facebook?, ¿tienes correo electrónico?, ¿tus compañeros tienen Facebook?, y si tienen para qué los usan?

Hija: si tengo Facebook, no tengo correo. Mis compañeros tienen Facebook la mayoría, lo usan para chatear con sus compañeros y no sé de qué hablan.

De la entrevista se evidencia que las TIC son usadas con fines particulares y que la incorporación de las mismas tiene poca incidencia en el proceso educativo. 


\subsection{Experiencia: I.E No 36344 de Callqui Chico-Chuñuranra}

El centro poblado de Chuñuranra está a $7 \mathrm{~km}$ de la ciudad de Huancavelica, una carretera transitable entre 10 a 15 minutos camino a la ciudad de Ica; sus comuneros se dedican a la ganadera de consumo y a la extracción de arena, por estar a orillas del río Ichu. La I.E N 36344 de Callqui Chico integra dos niveles educativos: Educación Inicial y Educación Primaria.

Los ambientes de la I.E son precarios. Cuenta con dos espacios dedicados a educación inicial: uno con material prefabricado, con techo de calamina y otro rústico (tapial y techo de calamina) (figuras 7 y 8 ).

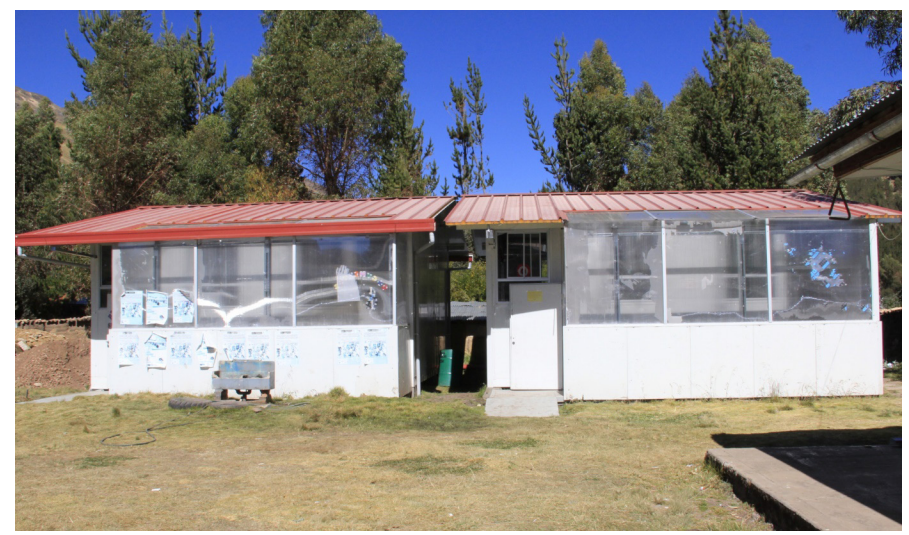

Figura 7. Edificio principal de la I.E N 36344 de Callqui ChicoChuñuranra.

Fuente: Archivo de los autores.

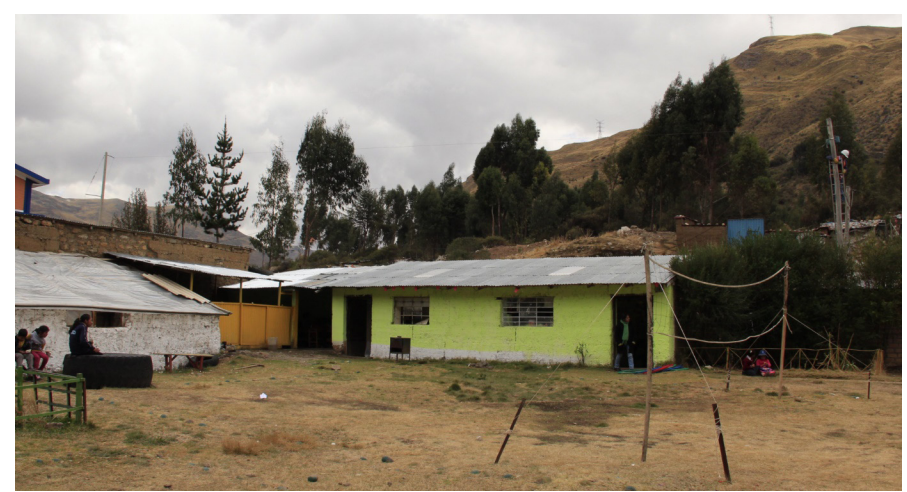

Figura 8. Edificio de apoyo de la I.E N 36344 de Callqui ChicoChuñuranra.

Fuente: Archivo de los autores. 
La infraestructura para educación primaria es de material rústico, construido con tapial y techo de calamina, cielorraso de triplay y piso de madera. Esta institución no cuenta con infraestructura deportiva. Esta actividad se realiza en el área libre de la institución (figura 9).

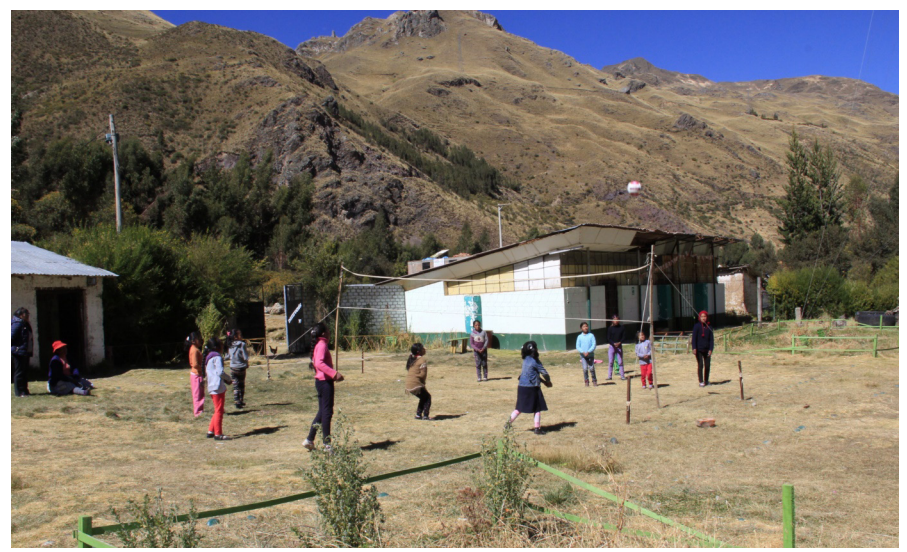

Figura 9. Predio para el recreo de la I.E N 36344 de Callqui Chico-Chuñuranra.

Fuente: Archivo de los autores.

Los servicios higiénicos son compartidos entre todos los integrantes de la comunidad educativa, generando problemas de higiene y salud (figura 10).

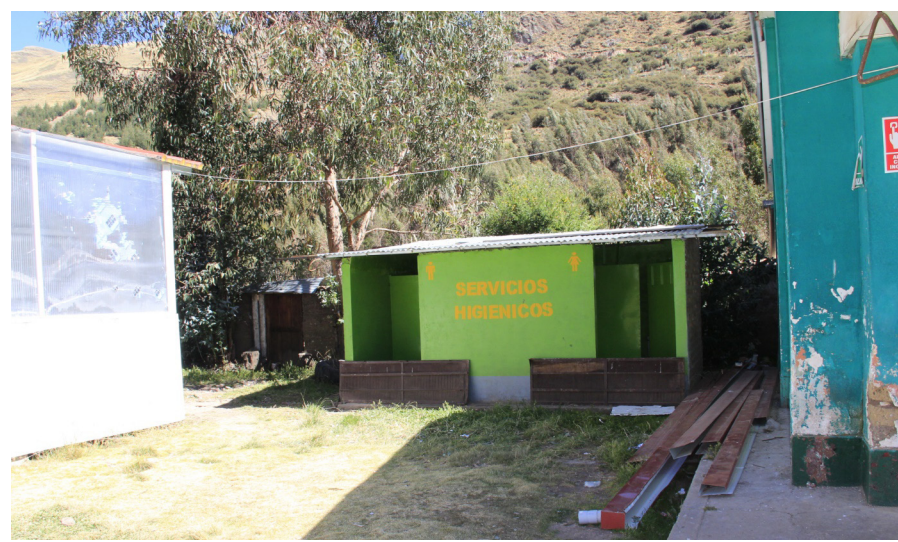

Figura 10. Servicios higiénicos de la I.E Nº36344 de Callqui Chico-Chuñuranra.

Fuente: Archivo de los autores.

Otro aspecto a destacar es que la institución educativa no cuenta con biblioteca. Los libros se encuentran almacenados en cajas y algunos en estantes (figura 11), limitando la posibilidad de consulta. 


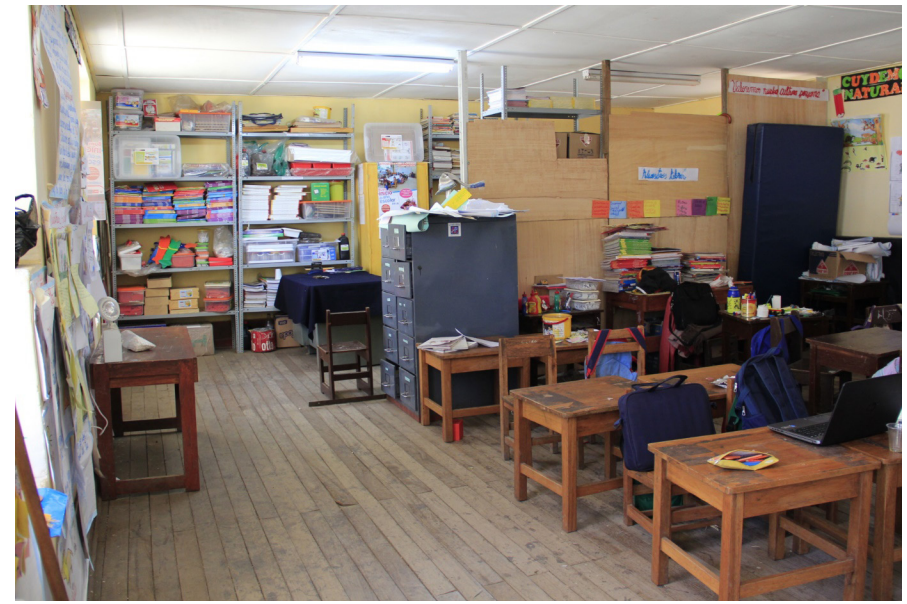

Figura 11. Material bibliográfico almacenado de la I.E Nº 36344 de Callqui Chico-Chuñuranra.

Fuente: Archivo de los autores.

\subsubsection{Las TIC en la Institución Educativa}

La I.E no evidencia el desarrollo de proyectos TIC orientados a las iniciativas propuestas por el MINEDU en cuanto a la incorporación de las TIC en los procesos educativos. Sin embargo, la DIGET, asume el compromiso en la capacitación de los docentes formadores y Docentes de Apoyo Tecnológico (DAT), a través de la plataforma PerúEduca, para la capacitación del SIAGIE offline y su complemento online.

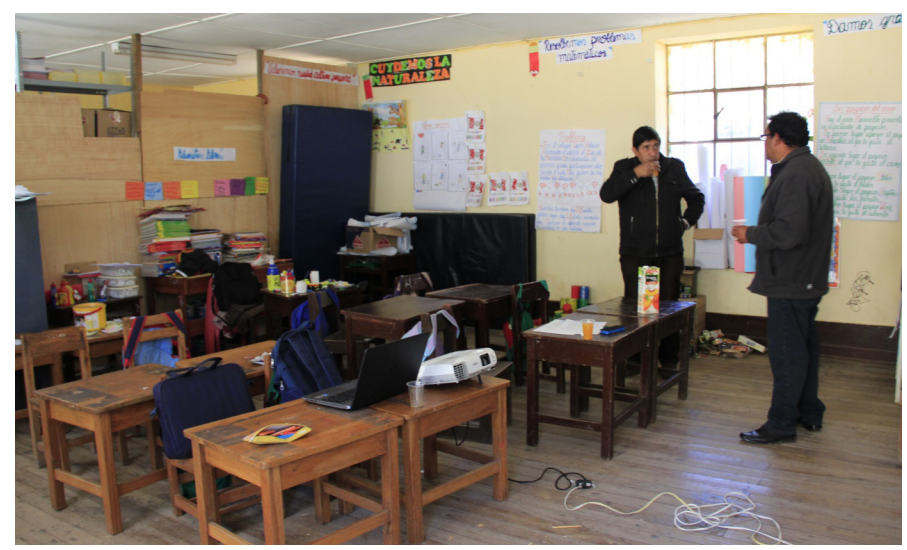

Figura 12. Aula común de la I.E N 36344 de Callqui ChicoChuñuranra.

Fuente: Archivo de los autores. 
La I.E cuenta con un número limitado de laptops XO (figura 12) y kits de robótica, almacenados en cajas; según apreciaciones del Director, su uso y manejo "quedó en intenciones". También es preciso indicar que, la I.E cuenta con internet de circuito cerrado del MINEDU y canal de televisión de PerúEduca, los que no son utilizados debido a que las horas programadas por el MINEDU no coindicen con el horario de los profesores.

\subsubsection{Las TIC y los profesores en el aula}

Los profesores de la I.E son siete en total, todos nombrados, cuyas edades oscilan entre 38 y 40 años. En palabras del Director, esta condición disminuye el interés por capacitarse y poner en práctica el uso de las TIC. A diferencia de los profesores, los estudiantes demuestran interés por tener acceso a las TIC, pero no existe aula de innovación tecnológica.

A pesar de las carencias tecnológicas los estudiantes poseen potencialidades, así lo expresa el director de la institución educativa:

... "ahora los profesores somos sólo facilitadores, pero todavía tenemos profesores que siguen escribiendo en la pizarra, mientras que los niños van a Internet donde recogen y procesan información" ... "Ios niños están ávidos en aprender todo sobre las TIC ... a veces traemos las XO ... están ya manipulando ingresando al Internet, los niños si tienen un gran interés, el problema que tenemos es que los profesores no están yendo al ritmo de las niños. Nos falta en si un aula de innovación para que los niños puedan aprender".

Similar caso sucede con el uso de los kits de robótica:

Director: ... "también tenemos el kit de robótica, pero aún ni yo y los profesores no sabemos utilizar, por lo que están guardados. En un momento intentamos llevar a cabo actividades con los niños, pero cuando quisimos insertarlo con las XO no pudimos hacer nada, ahí quedó porque no teníamos la orientación adecuada".

De la información emergente en las entrevistas pudimos conocer la debilidad en la adquisición de competencias en cuanto a las TIC por parte del personal docente.

\subsubsection{Participación de la familia en el uso de las TIC}

Prosiguiendo con los testimonios, el Director manifiesta "que existe poca participación de los padres de familia en la educación de sus hijos". Además, resalta en hecho de que "los niños trabajan para adquirir sus útiles escolares" 
De las entrevistas realizadas a padres de familia se evidencia que el $80 \%$ de hogares de Chuñuranra cuentan con, al menos, una tecnología de información y comunicación. El acceso a telefonía fija es muy limitado, mientras que la telefonía móvil va en incremento, alcanzando aproximadamente al $85 \%$ de la población en jóvenes edad promedio 16 años y con educación primaria completa. A la pregunta ¿Cuenta con teléfonos celulares en casa? Responden:

Madre 1: ... "rantinicu celularta tareancunata ruranankupaq, matemáticamanta, historiamanta y profesor saqesqanta".

Traducción: ... "compramos celulares para que hagan sus trabajos, de matemática, historia y lo que el profesor les asigna"

De las observaciones realizadas se desprende que los hogares que cuentan con una computadora alcanzan al 1,5\%; indican tenerla para uso exclusivo del hogar y trabajo sin acceso a internet. El acceso a internet es a través de cabinas públicas, aparte del indicado por los celulares, y se da más en hombres que en mujeres, población joven y adolescentes, puesto que tienen que movilizarse a la ciudad de Huancavelica.

A la pregunta ¿Cuenta con computadoras e Internet en casa?, los padres de familia responden:

Padre 1: ... "nosotros como gente pobre no podemos comprar las laptop ... una persona pobre que apenas tiene una chacrita, con eso estamos sobreviviendo, ... lo conveniente sería que en este colegio podrían proveernos como apoyo más laptop para nuestros hijos"

Padre 2: ... "para empezar, aquí en el colegio se ha creado el proyecto Huascarán, donde la APAFA, ha gestionado cinco computadoras implementadas con sistema de Internet inalámbrica y tuvimos una accesibilidad para nuestra juventud, el cual consideramos importante en ese tiempo, y ahora no se tiene"

Se constata el acceso a televisión por cable por un número considerable de hogares que dispone de este servicio, debido a que los canales de señal abierta no tienen cobertura en la zona.

A la interrogante ¿usted ayuda a sus hijos en las tareas escolares? Responde:

Madre 2: ... "ñoqaykuqa manan yachanikuchu ni yanapanikupas tareankunapiqa, hatun wawqenkunallam yachachinku sulkankunaman"

Traducción: ... "nosotras no sabemos ni les enseñamos las tareas, sólo sus hermanos mayores enseñan a los menores" 
Algunas familias han experimentado los beneficios de las TIC. Este resultado es producto de haber migrado a otros lugares por motivos de trabajo y han tenido experiencias con el uso de las tecnologías, así manifiesta un padre de familia:

Padre 3: ... "anteriormente han venido y empezaron con la tecnología de las laptop y las computadoras, el problema es que los docentes desconocían y no le enseñaban a los alumnos... comparando con los niños de cuarto grado de Huancavelica difieren; entonces, quiere decir que casi no le están enseñando en el dominio de la computadora...

... "la mayoría de aquí y nuestros comuneros nunca han salido hacia la ciudad, por eso no le dicen nada a sus hijos para que ellos sean profesionales; considero que hablándoles ya estamos promoviendo en ellos la idea de ser profesionales. Por ejemplo, las maquinarias son computarizadas y electrónicas; para reparar una retro excavadora, conectan una laptop y verifican donde se encuentra la falla; todas esas cosas deben enseñar en esta institución educativa. Ahora me siento alegre con la pronta llegada de la fibra óptica y la banda ancha, pediría mediante ustedes la mejora de nuestra institución educativa"

Se evidencia que los padres comprenden y valoran la importancia de las TIC en el proceso educativo, garantizando la adquisición de equipos dentro de sus posibilidades para que sus hijos realicen sus tareas académicas.

\section{A manera de conclusión}

Las experiencias presentadas en este estudio revelan que las instituciones educativas localizadas en zonas de pobreza y extrema pobreza manifiestan dificultades diversas para la puesta en marcha de iniciativas con TIC

Las políticas educativas diseñadas por el estado a través del MINEDU no han sido sostenibles en el tiempo, por lo que se ha mantenido inalterada la brecha digital existente en las instituciones educativas investigadas. Sería conveniente que las futuras políticas tomaran en consideración a los actores sociales, así como su entorno geográfico y socioeconómico. De esta forma se evitará inequidad y exclusión educativa de las poblaciones más vulnerables.

Se trata de un modelo 1 a 1 basado en una dotación que hubiese requerido del seguimiento por parte de la administración o la inspección educativa. También de la distribución y la implementación de infraestructura adecuada y módulos educativos. 
Por otra parte, los directores de las escuelas no parecen particularmente comprometidos con el impulso de proyectos en sus propios centros. La precariedad de los territorios en los que están enclavadas y las condiciones de pobreza de las familias deberían movilizar a los profesores para ofrecer alguna alternativa.

Los docentes no tienen preparación suficiente, pero tampoco hay voluntad de mejorar la capacitación o la reflexión sobre los usos de las TIC. La motivación y seguimiento de la capacitación docente en cuanto al uso de las TIC, en cada área o disciplina de conocimiento, es un elemento primordial a tomar en cuenta en la planificación para que estas políticas tengan los resultados esperados.

Otro hallazgo constituye la familia como actor principal en el proceso educativo, siendo necesaria la participación del niño y su familia en el uso de las TIC como herramienta pedagógica en articulación con los objetivos del currículo nacional.

Sin embargo, la presión de los padres en una de las escuelas y, paradójicamente, cierta voluntad de los docentes en ambas permite que los estudiantes manejen algunas tecnologías. En una de las escuelas imparten clases de Ofimática, pensando en el futuro de sus estudiantes como trabajadores. En la otra, los estudiantes piden a sus familias que les ayuden en sus tareas gracias a los teléfonos celulares, para completar tareas de clase. En los dos casos, las dotaciones tecnológicas no son las pensadas por las políticas oficiales, sino que provienen de una acción privada de las familias.

El aporte de investigaciones sobre la cosmovisión de los actores sociales del proceso educativo es relevante en el momento de establecer las orientaciones y planificación del uso de las TIC en las II.EE peruanas, conocer las necesidades de estos grupos de interés, estudiantes, profesores y la APAFA garantizará el desarrollo y sostenibilidad de estos procesos más allá de una visión asistencialista de la educación.

Hace falta aportar los elementos necesarios desde la investigación para elevar la calidad educativa y el nivel de vida de estos grupos sociales, a través del uso de las TIC, logrando la inclusión y equidad desde el enfoque 1 a 1.

\section{Agradecimiento}

Esta investigación fue posible gracia al proyecto "Exclusión, pobreza y TIC en las escuelas de América Latina y España", proyecto CEALAL/2015-31 financiado por la $9^{a}$ convocatoria de proyectos de cooperación Interuniversitaria UAM-Santander con América Latina. Resolución de 19 de 
junio de 2015. Asimismo, a Juana Luz Rivera Victoria y Oscar Sedano Chávez directora y profesor de la I.E José Antonio Encinas Franco de Llahuecc; a Alfredo Villanueva Ayala director de la I.E N 36344 de Callqui Chico-Chuñuranra; quienes brindaron información para la concreción del estudio.

\section{Referencias bibliográficas}

Area, M. (2011). Los efectos del modelo 1:1 en el cambio educativo en las escuelas. Evidencias y desafíos para las políticas iberoamericanas. Revista Iberoamericana de Educación, 56, 49-74. Recuperado de https://bit.ly/2tDcjHR

Balarín, M. (2013). Las políticas TIC en los sistemas educativos de América Latina: Caso Perú. Buenos Aires: Fondo de las Naciones Unidas para la Infancia (UNICEF). Recuperado de https://uni.cf/1RBAilm

Banco Mundial. (2015). Índice de Gini [Organización]. Recuperado de https://bit.ly/1psfn1c

Creswell, J. W. (2012). Qualitative Inquiry and Research Design: Choosing Among Five Approaches (3ra ed.). California: SAGE Publications.

El Peruano. (2016, febrero 23). Entregarán tabletas y kits de robótica. Se benefician colegios de 19 regiones. El Peruano / Diario Oficial, p. 11.

Expansión (2015). Gasto público Educación 2014 [Organismo]. Recuperado de https://bit. ly/2Xr9Xt5

INEI (2009). Perú: Tecnologías de Información y Comunicación en las empresas 2006 - 2007. LIma: Oficina Técnica de Administración (OTA) - INEI. Recuperado de https://bit. ly/2tD1Kob

INEI (2014) Perú: Indicadores de Educación por Departamentos, 2001-2012. Lima: Instituto Nacional de Estadística e Informática. Recuperado de https://bit.ly/2GMbC7s

INEI (2015a). Condiciones de vida en el Perú (Técnico No. 2) (p. 62). Lima: Instituto Nacional de Estadística e Informática. Recuperado de https://bit.ly/2T4YFfF

INEI (2015b). Día Mundial de la Población: Estado de la Población Peruana 2015. Instituto Nacional de Estadística e Informática. Recuperado de https://bit.ly/2av1D3g

INEI (2015c). Evolución de la pobreza monetaria 2009 - 2014 (Informe técnico). Lima: Instituto Nacional de Estadística e Informática (INEI). Recuperado de https://bit.ly/2VidzvK

INEI (2015d). PERU Instituto Nacional de Estadística e Informática INEI [Organización]. Recuperado de https://bit.ly/292cN1f

INEI (2018a). Evolución de la pobreza monetaria 2007 - 2017 (pp. 1-181). Lima: Instituto Nacional de Estadística e Informática. Recuperado de https://bit.ly/2rkWyEM

INEI (2018b). Perú: Perfil sociodemográfico. Censos Nacionales 2017: XII de Población, VII de vivienda y // de comunidades indíginas (Censos Nacionales 2017). Lima: Instituto Nacional de Estadística e Informática. Recuperado de https://bit.ly/2NZze8D

Jaureguiberry, F., López, Á. y Zoido, P. (2018, diciembre). ¿Cuánto invierten los gobiernos en educación? Centro de información para la mejora de los aprendizajes / BID. Recuperado de https://bit.ly/2NtrTyQ 
Linares, J. (2015). Robótica educativa en el Perú 1994 - 2014. Evaluaciones nacionales e internacionales en diferentes administraciones gubernamentales. Instituto de Tecnología Von Braun, (1), 1-29.

MINEDU (2001). Decreto Supremo N 067-2001-ED. Creación del Proyecto Huascarán. Recuperado de https://bit.ly/2Xsa9s2

MINEDU (2006). El proyecto Huascarán. La revista de educación “El Educador”, 2(7), 16-19.

Ñopo, H. (2018). Análisis de la inversión educativa en el Perú desde una mirada comparada (Proyecto FORGE No A-034597) (p. 36). Lima: GRADE. Recuperado de https:// bit.ly/2VoOP5d

OEA (2014). Desigualdad e inclusión social en las Américas. 14 ensayos (2da ed.). San Juan de Costa Rica: Washington. Recuperado de https://bit.ly/2VsaVUv

OEI (2016). Miradas sobre la Educación en Iberoamérica 2016. Avances en las Metas Educativas 2021. Madrid: Instituto de Evaluación (IESME) de la OEI. Recuperado de https:// bit.ly/2SslvZm

OLPC News (2011). El Futuro incierto del OLPC en el Perú. Recuperado de https://bit. Iy/2tFOEIE

OLPC News (2012). Entrevista con Sandro Marcone de OLPC Perú [Educación]. Recuperado de https://bit.ly/2U6vOnT

Peralta, J. I. y Lugo, M. T. (2016). Nuevas perspectivas educativas con TIC: caso Perú. En Entornos digitales y políticas educativas: dilemas y certezas. Buenos Aires: Instituto Internacional de Planeamiento de la Educación IIPE-Unesco. Recuperado de https:// bit.ly/2BTkhkx

Quintanilla, C., Cayllahua, U.y Canales, F. (2013). Las tecnologías de información y comunicación (TIC) en la formación del profesorado de matemática en educación secundaria. En A. Gewerc Barujel, E. Pernas Morado, A. Alonso Ferreiro y L. Montero Mesa (Eds.), I Congreso Internacional "Conocimiento, tecnologías y enseñanza: políticas y prácticas universitarias" (pp. 211-214). Santiago de Compostela: CONTECE 2013. Recuperado de https://bit.ly/2Xikl6u

Severin, E. y Capota, C. (2011). Modelos Uno a Uno en América Latina y el Caribe: Panorama y perspectivas. Banco Interamericano de Desarrollo. Recuperado de https://bit. Iy/2GZdyZT

SITEAL (2014). El Informe sobre Tendencias Sociales y Educativas en América Latina 2014. Políticas TIC en los sistemas educativos de América Latina. Buenos Aires: UNESCO. Recuperado de https://bit.ly/1Wily79

UNESCO (2015a). Educación 2030. Declaración de Incheon: Hacia una educación inclusiva y equitativa de calidad y un aprendizaje a lo largo de la vida para todos. UNESCO, París. Recuperado de https://bit.ly/2ejZBWa

UNESCO (2015b). Informe de Seguimiento de la EPT en el Mundo(p. 524). Francia. Recuperado de https://bit.ly/2rbhlZc

Vásquez, E. (2012). El Perú de los pobres no visibles para el Estado: La inclusión social pendiente a julio del 2012. Centro de Investigación de la Universidad del Pacífico. Recuperado de https://bit.ly/1EPnYQS

Webb, R. (2013). Concexión y despegue rural (Investigación) (p. 271). Lima: Universidad San Martín de Porres. Recuperado de https://bit.ly/2EdOAEL 